\title{
The Dichotomy for Conservative Constraint Satisfaction Problems Revisited
}

\author{
Libor Barto \\ Department of Mathematics and Statistics, McMaster University, Hamilton, ON, Canada \\ and \\ Department of Algebra, Charles University, Prague, Czech Republic \\ Email: libor.barto@gmail.com
}

\begin{abstract}
A central open question in the study of non-uniform constraint satisfaction problems (CSPs) is the dichotomy conjecture of Feder and Vardi stating that the CSP over a fixed constraint language is either NP-complete, or tractable. One of the main achievements in this direction is a result of Bulatov (LICS'03) confirming the dichotomy conjecture for conservative CSPs, that is, CSPs over constraint languages containing all unary relations. Unfortunately, the proof is very long and complicated, and therefore hard to understand even for a specialist. This paper provides a short and transparent proof.
\end{abstract}

\section{INTRODUCTION}

The constraint satisfaction problem (CSP) provides a common framework for many theoretical problems in computer science as well as for many real-life applications. An instance of the CSP consists of a number of variables and constraints imposed on them and the objective is to determine whether variables can be evaluated in such a way that all the constraints are met. The CSP can also be expressed as the problem of deciding whether a given conjunctive formula is satisfiable, or as the problem of deciding whether there exists a homomorphism between two relational structures.

The general CSP is NP-complete, however certain natural restrictions on the form of the constraints can ensure tractability. This paper deals with so called non-uniform CSP - the same decision problem as the ordinary CSP, but the set of allowed constraint relations is fixed. A central open problem in this area is the dichotomy conjecture of Feder and Vardi [1] stating that, for every finite, fixed set of constraint relations (a fixed constraint language), the CSP defined by it is NPcomplete or solvable in polynomial time, i.e. the class of CSPs exhibits a dichotomy.

Most of recent progress toward the dichotomy conjecture has been made using the algebraic approach to the CSP [2], [3], [4]. The main achievements include the algorithm for CSPs with "Maltsev constraints" [5] (which was substantially simplified in [6] and generalized in [7], [8]), the characterization of CSPs solvable by local consistency methods [9], [10], the dichotomy theorem for CSPs over a three element domain [11] (which generalizes the Boolean CSP dichotomy theorem [12]) and the dichotomy theorem for conservative CSPs [13].

The last result proves the dichotomy conjecture of Feder and Vardi for the CSP over any template which contains all unary relations. In other words, this Bulatov's theorem proves the dichotomy for the CSPs, in which we can restrict the value of each variable to an arbitrary subset of the domain (that is why the conservative CSPs are sometimes called list CSPs, or, in homomorphism setting, list homomorphism problems). This result is of major importance in the area, but, unfortunately, the proof is very involved (the full paper has 80 pages and it has not yet been published), which makes the study of possible generalizations and further research harder.

This paper provides a new, shorter and more natural proof. It relies on techniques developed and successfully applied in [14], [15], [16], [9], [17], [18].

\section{Related work}

The complexity of list homomorphism problems has been studied by combinatorial methods, e.g., in [19], [20]. A structural distinction between tractable and NP-complete list homomorphism problem for digraphs was found in [21]. A finer complexity classification for the list homomorphism problem for graphs was given in [22]. The conservative case is also studied for different variants of the CSP, see, e.g., [23], [24].

\section{Organization of the paper}

In Section I we define the CSP and its non-uniform version. In Section II we introduce the necessary notions concerning algebras and the algebraic approach to the CSP. In Section III we collect all the necessary ingredients. One of them is a reduction to minimal absorbing subuniverses, details are provided in Section V. Also the core algebraic result is just stated in this section and its proof covers Section VI. In Section IV we formulate the algorithm for tractable conservative CSPs and prove its correctness.

\section{CSP}

An $n$-ary relation on a set $A$ is a subset of the $n$-th cartesian power $A^{n}$ of the set $A$.

Definition I.1. $A n$ instance of the constraint satisfaction problem $(C S P)$ is a triple $P=(V, A, \mathcal{C})$ with

- $V$ a nonempty, finite set of variables,

- A a nonempty, finite domain,

- $\mathcal{C}$ a finite set of constraints, where each constraint is a pair $C=(\mathbf{x}, R)$ with 
- $\mathrm{x}$ a tuple of distinct variables of length $n$, called the scope of $C$, and

- $R$ an $n$-ary relation on $A$, called the constraint relation of $C$.

The question is whether there exists a solution to $P$, that is, a function $f: V \rightarrow A$ such that, for each constraint $C=(\mathbf{x}, R) \in \mathcal{C}$, the tuple $f(\mathbf{x})$ belongs to $R$.

For purely technical reasons we have made a nonstandard assumption that the scope of a constraint contains distinct variables. This clearly does not change the complexity modulo polynomial-time reductions.

In the non-uniform CSP we fix a domain and a set of allowed constraints:

Definition I.2. A constraint language $\Gamma$ is a set of relations on a finite set $A$. The constraint satisfaction problem over $\Gamma$, denoted $\operatorname{CSP}(\Gamma)$, is the subclass of the CSP defined by the property that any constraint relation in any instance must belong to $\Gamma$.

The following dichotomy conjecture was originally formulated in [1] only for finite constraint languages. The known results suggest that even the following stronger version might be true.

Conjecture I.3. For every constraint language $\Gamma, \operatorname{CSP}(\Gamma)$ is either tractable, or NP-complete.

Our main theorem, first proved by Bulatov [13], confirms the dichotomy conjecture for conservative CSPs:

Definition I.4. A constraint language $\Gamma$ on $A$ is called conservative, if $\Gamma$ contains all unary relations on $A$ (i.e., all subsets of $A$ ).

Theorem I.5. For every conservative constraint language $\Gamma$, $\operatorname{CSP}(\Gamma)$ is either tractable, or NP-complete.

\section{AlgEBRA AND CSP}

\section{A. Algebraic preliminaries}

An $n$-ary operation on a set $A$ is a mapping $f: A^{n} \rightarrow A$. An operation $f$ is called cyclic, if $n \geq 2$ and $f\left(a_{1}, a_{2}, \ldots, a_{n}\right)=$ $f\left(a_{2}, a_{3}, \ldots, a_{n}, a_{1}\right)$ for any $a_{1}, a_{2}, \ldots, a_{n} \in A$. A ternary operation $m$ is called Maltsev, if $f(a, a, b)=f(b, a, a)=b$ for any $a, b \in A$.

A signature is a finite set of symbols with natural numbers (the arities) assigned to them. An algebra of a signature $\Sigma$ is a pair $\mathbf{A}=\left(A,\left(t^{\mathbf{A}}\right)_{t \in \Sigma}\right)$, where $A$ is a set, called the universe of $\mathbf{A}$, and $t^{\mathbf{A}}$ is an operation on $A$ of arity $\operatorname{ar}(t)$. We use a boldface letter to denote an algebra and the same letter in the plain type to denote its universe. We omit the superscripts of operations as the algebra is always clear from the context.

A term operation of $\mathbf{A}$ is an operation which can be obtained from operations in $\mathbf{A}$ using composition and the projection operations. The set of all term operations of $\mathbf{A}$ is denoted by $\mathrm{Clo}(\mathbf{A})$.

There are three fundamental operations on algebras of a fixed signature $\Sigma$ : forming subalgebras, factoralgebras and products.
A subset $B$ of the universe of an algebra $\mathbf{A}$ is called a subuniverse, if it is closed under all operations (equivalently term operations) of $\mathbf{A}$. Given a subuniverse $B$ of $\mathbf{A}$ we can form the algebra $\mathbf{B}$ by restricting all the operations of $\mathbf{A}$ to the set $B$. In this situation we say that $\mathbf{B}$ is a subalgebra of $\mathbf{A}$ and we write $B \leq \mathbf{A}$ or $\mathbf{B} \leq \mathbf{A}$. We call the subuniverse $B$ (or the subalgebra $\mathbf{B}$ ) proper if $\emptyset \neq B \neq A$.

We define the product of algebras $\mathbf{A}_{1}, \ldots, \mathbf{A}_{n}$ to be the algebra with the universe equal to $A_{1} \times \cdots \times A_{n}$ and with operations computed coordinatewise. The product of $n$ copies of an algebra $\mathbf{A}$ is denoted by $\mathbf{A}^{n}$.

An equivalence relation $\sim$ on the universe of an algebra $\mathbf{A}$ is a congruence, if it is a subalgebra of $\mathbf{A}^{2}$. The corresponding factor algebra $\mathbf{A} / \sim$ has, as its universe, the set of $\sim$ blocks and operations are defined using (arbitrary chosen) representatives. Every algebra $\mathbf{A}$ has two trivial congruences: the diagonal congruence $\sim=\{(a, a): a \in A\}$ and the full congruence $\sim=A \times A$. A congruence is proper, if it is not equal to the full congruence. A congruence is maximal, if the only coarser congruence of $\mathbf{A}$ is the full congruence.

For a finite algebra $\mathbf{A}$ the class of all factor algebras of subalgebras of finite powers of $\mathbf{A}$ will be denoted by $\mathrm{V}_{\text {fin }}(\mathbf{A})$.

An operation $f: A^{n} \rightarrow A$ is idempotent, if $f(a, a, \ldots, a)=$ $a$ for any $a \in A$. An operation $f: A^{n} \rightarrow A$ is conservative, if $f\left(a_{1}, a_{2}, \ldots, a_{n}\right) \in\left\{a_{1}, a_{2}, \ldots, a_{n}\right\}$ for any $a_{1}, a_{2}, \ldots, a_{n} \in$ $A$. An algebra is idempotent (resp. conservative), if all operations of $\mathbf{A}$ are idempotent (resp. conservative). In other words, an algebra is idempotent (resp. conservative), if all oneelement subsets of $A$ (resp. all subsets of $A$ ) are subuniverses of $\mathbf{A}$.

\section{B. Algebraic approach}

An operation $f: A^{n} \rightarrow A$ is compatible with a relation $R \subseteq$ $A^{m}$ if the tuple

$\left(f\left(a_{1}^{1}, a_{1}^{2}, \ldots, a_{1}^{n}\right), f\left(a_{2}^{1}, a_{2}^{2}, \ldots, a_{2}^{n}\right), \ldots, f\left(a_{m}^{1}, a_{m}^{2}, \ldots, a_{m}^{n}\right)\right.$

belongs to $R$ whenever $\left(a_{1}^{i}, a_{2}^{i}, \ldots, a_{m}^{i}\right) \in R$ for all $i \leq n$.

An operation compatible with all relations in a constraint language $\Gamma$ is a polymorphism of $\Gamma$. The set $A$ together with all polymorphisms of $\Gamma$ is the algebra of polymorphisms of $\Gamma$, it is denoted Pol $\Gamma$, or often just $\mathbf{A}$ (we formally define the signature of $\mathbf{A}$ to be identical with the set of its operations). Note that every relation in $\Gamma$ is a subalgebra of a power of $\mathbf{A}$. The set of all subalgebras of powers of $\mathbf{A}$ is denoted by Inv A.

In the following discussion we assume, for simplicity, that $\Gamma$ contains all singleton unary relations (it is known that CSP can be reduced to CSP over such a constraint language). Observe that in such a case the algebra $\mathbf{A}$ is idempotent. Moreover, if $\Gamma$ is conservative, then $\mathbf{A}$ is conservative as well.

Already the first results on the algebraic approach to CSP [2], [3], [4] show that A fully determines the computational complexity of $\operatorname{CSP}(\Gamma)$, at least for finite constraint languages. Moreover, a borderline between tractable and NPcomplete CSPs was conjectured in terms of the algebra of polymorphisms: if there exists a two-element factor algebra 
of a subalgebra of $\mathbf{A}$ whose every operation is a projection, then $\operatorname{CSP}(\Gamma)$ is NP-complete, otherwise $\operatorname{CSP}(\Gamma)$ is tractable. The hardness part of this algebraic dichotomy conjecture is known [3], [4]:

Theorem II.1. Let $\Gamma$ be a constraint language containing all singleton unary relations, and let $\mathbf{A}=\mathrm{Pol} \Gamma$. If $\mathbf{A}$ has a subalgebra with a two-element factor algebra whose every operation is a projection, then $\operatorname{CSP}(\Gamma)$ is NP-complete.

The algebras, which satisfy this necessary (and conjecturally sufficient) condition for tractability, are called Taylor algebras, that is, $\mathbf{A}$ is Taylor if no two-element factor algebra of a subalgebra $\mathbf{A}$ has projection operations only. We will use the following characterization of Taylor algebras from [17], [18] although the characterization in terms of weak near-unanimity operations [25] would suffice for our purposes.

Theorem II.2. Let $\mathbf{A}$ be a finite idempotent algebra and let $p>|A|$ be a prime number. The following are equivalent.

- A is a Taylor algebra.

- A has a cyclic term operation of arity p.

In view of Theorem II.1, the dichotomy for conservative CSPs will follow when we prove:

Theorem II.3. Let $\mathbf{A}$ be a finite conservative Taylor algebra. Then $\operatorname{CSP}(\operatorname{Inv} \mathbf{A})$ is tractable.

A polynomial time algorithm for solving $\operatorname{CSP}(\operatorname{Inv} \mathbf{A})$, where $\mathbf{A}$ is a finite conservative Taylor algebra, is presented in Section IV.

\section{INGREDIENTS}

The building blocks of our algorithm are the $(k, l)$-minimality algorithm (Subsection III-B), a reduction to minimal absorbing subuniverses (Subsection III-D) and the algorithm for Maltsev instances (Subsection III-F). Subsection III-A and Subsection III-C cover necessary notation. The main new algebraic tool for proving correctness is stated in Subsection III-E.

\section{A. Projections and restrictions}

Tuples are denoted by boldface letters and their elements are indexed from 1 , for instance $\mathbf{a}=\left(a_{1}, a_{2}, \ldots, a_{n}\right)$. For an $n$-tuple $\mathbf{a}$ and a tuple $\mathbf{k}=\left(k_{1}, \ldots, k_{m}\right)$ of elements of $\{1,2, \ldots, n\}$ we define the projection of $\mathbf{a}$ to $\mathbf{k}$ by

$$
\left.\mathbf{a}\right|_{\mathbf{k}}=\left(a_{k_{1}}, a_{k_{2}}, \ldots, a_{k_{m}}\right) .
$$

For a subset $K \subseteq\{1,2, \ldots, n\}$ we put $\left.\mathbf{a}\right|_{K}=\left.\mathbf{a}\right|_{\mathbf{k}}$, where $\mathbf{k}$ is the list of elements of $K$ in the ascending order.

The projection of a set $R \subseteq A_{1} \times \ldots A_{n}$ to $\mathbf{k}$ (resp. $K$ ) is defined by

$$
\left.R\right|_{\mathbf{k}}=\left\{\left.\mathbf{a}\right|_{\mathbf{k}}: \mathbf{a} \in R\right\} \quad\left(\text { resp. }\left.R\right|_{K}=\left\{\left.\mathbf{a}\right|_{K}: \mathbf{a} \in R\right\}\right) .
$$

The set $R$ is subdirect in $A_{1} \times \cdots \times A_{n}$ (denoted by $R \subseteq_{S}$ $\left.A_{1} \times \cdots \times A_{n}\right)$, if $\left.R\right|_{\{i\}}=A_{i}$ for all $i=1, \ldots, n$. If, moreover, $\mathbf{A}_{1}, \ldots, \mathbf{A}_{n}$ are algebras of the same signature and $R$ is a subalgebra of their product, we write $R \leq_{S} \mathbf{A}_{1} \times \cdots \times \mathbf{A}_{n}$.
Let $P=(V, A, \mathcal{C})$ be an instance of the CSP. The projection of a constraint $C=\left(\left(x_{1}, \ldots, x_{n}\right), R\right) \in \mathcal{C}$ to a tuple of variables $\left(x_{k_{1}}, \ldots, x_{k_{m}}\right)$ is the relation

$$
\left.C\right|_{\left(x_{k_{1}}, \ldots, x_{k_{m}}\right)}=\left\{\left(a_{k_{1}}, \ldots, a_{k_{m}}\right): \mathbf{a} \in R\right\} .
$$

Finally, we introduce two types of restrictions of a CSP instance. In the variable restriction we delete some of the variables and replace the constraints with appropriate projections, in the domain restriction we restrict the value of some of the variables to specified subsets of the domain.

The variable restriction of $P$ to a subset $W \subseteq V$ is the instance $\left.P\right|_{W}=\left(W, A, \mathcal{C}^{\prime}\right)$, where $\mathcal{C}^{\prime}$ is obtained from $\mathcal{C}$ by replacing each constraint $C=(\mathrm{x}, R) \in \mathcal{C}$ with $\left(\mathbf{x} \cap W,\left.C\right|_{\mathbf{x} \cap W}\right)$, where $\mathbf{x} \cap W$ is the subtuple of $\mathbf{x}$ formed by the variables belonging to $W$.

The domain restriction of $P$ to a system $\mathcal{E}=\left\{E_{x}\right.$ : $x \in W\}$ of subsets of $A$ indexed by $W \subseteq V$ is the instance $\left.P\right|_{\mathcal{E}}=\left(V, A, \mathcal{C}^{\prime}\right)$, where $\mathcal{C}^{\prime}$ is obtained from $\mathcal{C}$ by replacing each constraint $C=\left(\left(x_{1}, \ldots, x_{n}\right), R\right) \in \mathcal{C}$ with $C^{\prime}=\left(\left(x_{1}, \ldots, x_{n}\right),\left\{\mathbf{a} \in R: \forall i x_{i} \in W \Rightarrow a_{i} \in E_{x_{i}}\right\}\right)$.

\section{B. $(k, l)$-minimality}

The first step in our algorithm will be to ensure a certain kind of local consistency. The following notion is the most convenient for our purposes.

Definition III.1. Let $l \geq k>0$ be natural numbers. An instance $P=(V, A, \mathcal{C})$ of the $C S P$ is $(k, l)$-minimal, if:

- Every at most l-element tuple of distinct variables is the scope of some constraint in $\mathcal{C}$,

- For every tuple $\mathrm{x}$ of at most $k$ variables and every pair of constraints $C_{1}$ and $C_{2}$ from $\mathcal{C}$ whose scopes contain all variables from $\mathrm{x}$, the projections of the constraints $C_{1}$ and $C_{2}$ to $\mathrm{x}$ are the same.

$A(k, k)$-minimal instance is also called $k$-minimal.

For fixed $k, l$ there is an obvious polynomial time algorithm for transforming an instance of the CSP to a $(k, l)$-minimal instance with the same set of solutions: First we add dummy constraints to ensure that the first condition is satisfied and then we gradually remove those tuples from the constraint relations which falsify the second condition (see [13] for a more detailed discussion). It is a folklore fact (which is in the literature often used without mentioning) that an instance of $\operatorname{CSP}(\operatorname{Inv} \mathbf{A})$ is in this way transformed to an instance of $\operatorname{CSP}(\operatorname{Inv} \mathbf{A})$, that is, the constraint relations of the new instance are still members of Inv A. See the discussion after Definition III.3 in [9], where an argument is given for a similar consistency notion.

If an instance $P$ is (at least) 1-minimal, then, for each variable $x \in V$, there is a unique constraint whose scope is $(x)$. We denote its constraint relation by $S_{x}^{P}$, i.e. $\left((x), S_{x}^{P}\right) \in \mathcal{C}$. Then the projection of any constraint whose scope contains $x$ to $(x)$ is equal to $S_{x}^{P}$. If, moreover, $P$ is an instance of $\operatorname{CSP}(\operatorname{Inv} \mathbf{A})$, the set $S_{x}^{P}$ is a subuniverse of $\mathbf{A}$ and we denote the corresponding subalgebra of $\mathbf{A}$ by $\mathbf{S}_{x}^{P}$.

If an instance is 2-minimal, then we have a unique constraint $\left(\left(x, x^{\prime}\right), S_{\left(x, x^{\prime}\right)}^{P}\right)$ for each pair of distinct variables $x, x^{\prime} \in V$, 
and $\left.C\right|_{\left(x, x^{\prime}\right)}=S_{\left(x, x^{\prime}\right)}^{P}$ for any constraint $C$ whose scope contains $x$ and $x^{\prime}$. We formally define $S_{(x, x)}^{P}=\left\{(a, a): a \in S_{x}^{P}\right\}$.

$(2,3)$-minimal instances have the following useful property.

Lemma III.2. Let $P$ be a (2,3)-minimal instance and let $x, x^{\prime}, x^{\prime \prime} \in V$. Then for any $\left(a, a^{\prime}\right) \in S_{\left(x, x^{\prime}\right)}^{P}$ there exists $a^{\prime \prime} \in$ $A$ such that $\left(a, a^{\prime \prime}\right) \in S_{\left(x, x^{\prime \prime}\right)}^{P}$ and $\left(a^{\prime}, a^{\prime \prime}\right) \in S_{\left(x^{\prime}, x^{\prime \prime}\right)}^{P}$.

Proof: Let $C \in \mathcal{C}$ be a constraint with the scope $\left(x, x^{\prime}, x^{\prime \prime}\right)$, say $C=\left(\left(x, x^{\prime}, x^{\prime \prime}\right), R\right)$. The projection of $C$ to $\left(x, x^{\prime}\right)$ is equal to $S_{\left(x, x^{\prime}\right)}^{P}$, therefore there exists $a^{\prime \prime} \in A$ such that $\left(a, a^{\prime}, a^{\prime \prime}\right) \in R$. This element satisfies the conclusion of the lemma.

\section{Walking with subsets}

Let $R \subseteq A_{1} \times A_{2}$ and let $B \subseteq A_{1}$. We define

$$
R^{+}[B]=\left\{c \in A_{2}: \exists b \in B \quad(b, c) \in R\right\} .
$$

A qoset is a set $A$ together with a quasi-ordering on $A$, i.e. a reflexive, transitive (binary) relation $\leq$ on $A$. The blocks of the induced equivalence $\sim$, given by $a \sim b$ iff $a \leq b \leq a$, are called components of the qoset. A component $C$ is maximal, if $a \sim c$ for any $a \in A, c \in C$ such that $c \leq a$. A subqoset is a subset of $A$ together with $\leq$ restricted to $A$.

For a 1-minimal instance $P=(V, A, \mathcal{C})$ we introduce a qoset $\operatorname{Qoset}(P)$ as follows. The elements are all the pairs $(x, B)$, where $x \in V$ and $B$ is a subset of $S_{x}^{P}$. We put $(x, B) \leq\left(x^{\prime}, B^{\prime}\right)$, if there exists a constraint $C \in \mathcal{C}$ whose scope contains $\left\{x, x^{\prime}\right\}$ such that $\left.C\right|_{\left(x, x^{\prime}\right)}{ }^{+}[B]=B^{\prime}$. The ordering of the qoset $\operatorname{Qoset}(P)$ is the transitive closure of $\leq$.

If the instance $P$ is $(2,3)$-minimal, the components of Qoset $(P)$ are nicely behaved:

Proposition III.3. Let $P=(V, A, \mathcal{C})$ be a $(2,3)$-minimal instance of the CSP and let $(x, B)$ and $\left(x^{\prime}, B^{\prime}\right)$ be two elements of the same component of the qoset $\operatorname{Qoset}(P)$. Then $S_{\left(x, x^{\prime}\right)}^{P}{ }^{+}[B]=B^{\prime}$. In particular, if $x=x^{\prime}$, then $B=B^{\prime}$.

Proof: Let $(x, B)=\left(x_{1}, B_{1}\right),\left(x_{2}, B_{2}\right), \ldots,\left(x_{k}, B_{k}\right)=$ $\left(x^{\prime}, B^{\prime}\right)$ be a sequence of elements of $\operatorname{Qoset}(P)$ such that $S_{\left(x_{i}, x_{i+1}\right)}^{P}\left[B_{i}\right]=B_{i+1}$ for all $i=1, \ldots, k-1$. From Lemma III.2 it follows that

$$
S_{\left(x_{1}, x_{i+1}\right)}^{P}{ }^{+}\left[B_{1}\right] \subseteq S_{\left(x_{i}, x_{i+1}\right)}^{P}+\left[S_{\left(x_{1}, x_{i}\right)}^{P}{ }^{+}\left[B_{1}\right]\right],
$$

therefore $S_{\left(x, x^{\prime}\right)}^{P}{ }^{+}[B] \subseteq B^{\prime}$. Similarly, $S_{\left(x^{\prime}, x\right)}^{P}{ }^{+}\left[B^{\prime}\right] \subseteq B$. For each $b^{\prime} \in B^{\prime}\left(\subseteq S_{x^{\prime}}^{P}\right)$ there exists $b \in A$ such that $\left(b, b^{\prime}\right) \in S_{\left(x, x^{\prime}\right)}^{P}{ }^{+}[B]$. This element $b$ has to belong to $B$ (since $\left.S_{\left(x^{\prime}, x\right)}^{P}{ }^{+}\left[B^{\prime}\right] \subseteq B\right)$, which proves the inclusion $S_{\left(x, x^{\prime}\right)}^{P}{ }^{+}[B] \supseteq$ $B^{\prime}$.

Let $P$ be a $(2,3)$-minimal instance and $\mathcal{E}=\left\{E_{x}: x \in V\right\}$ be a system of subsets of $A$ such that $E_{x} \subseteq S_{x}^{P}$ for each $x \in V$. A $(P, \mathcal{E})$-strand is a maximal subset $W$ of $V$ such that all the pairs $\left(x, E_{x}\right), x \in W$ belong to the same component of the qoset $\operatorname{Qoset}(P)$. The name of this concept is justified by the previous proposition: For example, any solution $f: V \rightarrow A$ to $P$ with $f(x) \in E_{x}$ for some $x \in W$ satisfies $f(x) \in E_{x}$ for all $x \in W$.

\section{Absorbing subuniverses}

Definition III.4. Let $\mathbf{A}$ be a finite idempotent algebra and $t \in \mathrm{Clo}(\mathbf{A})$. We say that a subalgebra $\mathbf{B}$ of $\mathbf{A}$ is an absorbing subalgebra of $\mathbf{A}$ with respect to $t$ if, for any $k \leq \operatorname{ar}(t)$, any choice of $a_{i} \in A$ such that $a_{i} \in B$ for all $i \neq k$ we have $t\left(a_{1}, \ldots, a_{\operatorname{ar}(t)}\right) \in B$.

We say that $\mathbf{B}$ is an absorbing subalgebra of $\mathbf{A}$, or that $\mathbf{B}$ absorbs $\mathbf{A}$ (and write $\mathbf{B} \triangleleft \mathbf{A}$ ), if there exists $t \in \mathrm{Clo}(\mathbf{A})$ such that $\mathbf{B}$ is an absorbing subalgebra of $\mathbf{A}$ with respect to $t$.

We say that $\mathbf{A}$ is an absorption free algebra, if it has no proper absorbing subalgebras.

We also speak about absorbing subuniverses i.e. universes of absorbing subalgebras.

Definition III.5. If $\mathbf{B} \triangleleft \mathbf{A}$ and no proper subalgebra of $\mathbf{B}$ absorbs $\mathbf{A}$, we call $\mathbf{B}$ a minimal absorbing subalgebra of $\mathbf{A}$ (and write $\mathbf{B} \triangleleft \triangleleft \mathbf{A}$ ).

Alternatively, we can say that $\mathbf{B}$ is a minimal absorbing subalgebra of $\mathbf{A}$, if $\mathbf{B} \triangleleft \mathbf{A}$ and $\mathbf{B}$ is an absorption free algebra. Equivalence of these definitions follows from transitivity of $\triangleleft$ (see Proposition III.2 in [17]).

Algorithm 1 finds, for a given $(2,3)$-minimal instance $P$ of the CSP, a domain restriction $Q$ of $P$ which is 1-minimal and satisfies $\mathbf{S}_{x}^{Q} \triangleleft \triangleleft \mathbf{S}_{x}^{P}$ for any $x \in V$.

The algorithms uses a subqoset AbsQoset $(P)$ of $\operatorname{Qoset}(P)$ formed by the elements $(x, B)$ such that $B$ is a proper absorbing subuniverse of $\mathbf{S}_{x}^{P}$.

Fig. 1. Algorithm 1: Minimal absorbing subuniverses

Input: $(2,3)$-minimal instance $P=(V, A, \mathcal{C})$ of $\operatorname{CSP}(\operatorname{Inv} \mathbf{A})$

Output: $\mathcal{E}=\left\{E_{x}: x \in V\right\}$ such that $E_{x} \triangleleft \triangleleft \mathbf{S}_{x}^{P}, x \in V$, and $\left.P\right|_{\mathcal{E}}$ is 1-minimal

1: while some $S_{x}^{P}$ has a proper absorbing subuniverse do

2: find a maximal component $\mathcal{F}=\left\{\left(x, E_{x}\right): x \in W\right\}$ of the qoset AbsQoset $(P)$

3: $\quad P:=\left.P\right|_{\mathcal{F}}$

4: return $\left\{S_{x}^{P}: x \in V\right\}$

Theorem III.6. Algorithm 1 is correct and, for a fixed idempotent algebra $\mathbf{A}$, works in polynomial time.

Proof: The qoset AbsQoset $(P)$ contains at most $2^{|A|}|V|$ elements, therefore its maximal component can be found in a polynomial time. In each while loop at least one of the sets $S_{x}^{P}$ becomes smaller, thus the while loop is repeated at most $|V||A|$ times, and the algorithm is therefore polynomial.

The correctness follows from a slightly generalized results from [9] (the generalized version will be in [10]): In the beginning of the while loop, $P$ is so called Prague strategy. An analogue of Proposition III.3 remains valid for Prague strategies (Lemma IV.10 in [9], Lemma V.5 part (iii) in Section $\mathrm{V})$, in particular, for each variable $x \in V$, there is at most 
one element $\left(x, E_{x}\right)$ in the maximal component, therefore the definition of $\mathcal{F}$ in step 2 makes sense. Finally, the restriction of $P$ to $\mathcal{F}$ is again a Prague strategy (Theorem IV.15 in [9], Lemma V.6 in Section V). The details are in Section V.

\section{E. Rectangularity}

The core result for proving correctness of our algorithm for conservative CSPs is the "Rectangularity Theorem". We state the theorem here, its proof spans Section VI.

We need one more notion. Let $A_{1}, \ldots, A_{n}, B_{1}, \ldots, B_{n}$ be sets such that $B_{i} \subseteq A_{i}$ and let $R \subseteq_{S} A_{1} \times \cdots \times A_{n}$. We define a quasi-ordering $\preceq$ on the set $\{1,2, \ldots, n\}$ by

$$
i \preceq j \quad \text { if }\left.\quad R\right|_{(i, j)}{ }^{+}\left[B_{i}\right] \subseteq B_{j} .
$$

Components of this qoset are called $(R, B)$-strands.

Theorem III.7. Let $\mathbf{A}$ be a finite Taylor algebra, let $\mathbf{A}_{1}, \ldots, \mathbf{A}_{n}, \mathbf{B}_{1}, \ldots, \mathbf{B}_{n} \in \mathrm{V}_{\mathrm{fin}}(\mathbf{A})$ be conservative algebras such that $\mathbf{B}_{i} \triangleleft \triangleleft \mathbf{A}_{i}$ for all $i$, let $R \leq_{S} \mathbf{A}_{1} \times \cdots \times \mathbf{A}_{n}$ and assume that $R \cap\left(B_{1} \times \ldots, B_{n}\right) \neq \emptyset$. Then a tuple $\mathbf{a} \in B_{1} \times \cdots \times B_{n}$ belongs to $R$ whenever $\left.\left.\mathbf{a}\right|_{K} \in R\right|_{K}$ for each $(R, B)$-strand $K$.

\section{F. Maltsev instances}

Our final ingredient is the polynomial time algorithm by $\mathrm{Bu}$ latov and Dalmau [6] for the CSPs over constraint languages with a Maltsev polymorphism. Their algorithm can be used without any change in the following setting:

Theorem III.8. [6] Let $\mathbf{A}$ be a finite algebra with a ternary term operation $m$. Then there is a polynomial time algorithm which correctly decides every 1-minimal instance $P$ of $\mathrm{CSP}(\operatorname{Inv} \mathbf{A})$ such that, for every variable $x, m$ is a Maltsev operation of $\mathbf{S}_{x}^{P}$.

\section{Algorithm}

The algorithm for conservative CSPs is in Figure 2. It uses a subqoset NafaQoset $(P)$ of the qoset $\operatorname{Qoset}(P)$ formed by the elements $(x, B)$ such that $B \subseteq S_{x}^{P}$ and $\mathbf{B}$ has a proper absorbing subalgebra (where $\mathbf{B}$ stands for the subalgebra of A with universe $B$ ).

Theorem IV.1. If $\mathbf{A}$ is a conservative finite algebra, then Algorithm 2 is correct and works in polynomial time.

Proof: By induction on $k$ we show that the algorithm works in polynomial time for all instances such that $\left|S_{x}^{P}\right| \leq k$. The base case of the induction is obvious: if every $S_{x}^{P}$ is at most one-element, then the algorithm proceeds directly to Step 15 (where the algorithm answers YES iff every $S_{x}^{P}$ is one-element).

Step 1 can be done in polynomial time as discussed in Subsection III-B. In Step 3 the qoset has size at most $2^{|A|}|V|$, therefore its maximal component can be found in polynomial time. Step 5 is polynomial according to Theorem III.6. There are at most $|V|$ repetitions of the for cycle in Step 6. Step 7 is polynomial by the induction hypothesis, since every $E_{x}$ is a minimal absorbing subuniverse of $\mathbf{D}_{x}$ and $\mathbf{D}_{x}$ has, as a
Fig. 2. Algorithm 2 for solving $C S P(\operatorname{Inv} \mathbf{A})$ for conservative $\mathbf{A}$ Input: Instance $P=(V, A, \mathcal{C})$ of $\operatorname{CSP}(\operatorname{Inv} \mathbf{A})$

Output: "YES" if $P$ has a solution, "NO" otherwise

1: Transform $P$ to a $(2,3)$-minimal instance with the same solution set

2: if some subalgebra of $S_{x}^{P}$ has a proper absorbing subalgebra then

3: $\quad$ Find a maximal component $\mathcal{D}=\left\{D_{x}: x \in W\right\}$ of NafaQoset $(P)$

4: $\quad Q:=\left.\left(\left.P\right|_{W}\right)\right|_{\mathcal{D}}$

5: $\quad \mathcal{E}:=$ the result of Algorithm 1 for the instance $Q$

for each $(Q, \mathcal{E})$-strand $U$ do

Use this algorithm for the instance $\left.\left(\left.Q\right|_{U}\right)\right|_{\left\{E_{x}: x \in U\right\}}$

if no solution exists then

$$
\mathcal{F}:=\left\{S_{x}^{P}-E_{x}: x \in U\right\}
$$$$
P:=\left.P\right|_{\mathcal{F}}
$$$$
\text { goto step } 1
$$

$\mathcal{F}:=\left\{S_{x}^{P}-\left(D_{x}-E_{x}\right): x \in W\right\}$

$P:=\left.P\right|_{\mathcal{F}}$

goto step 1

15: Use the algorithm for Maltsev instances (Theorem III.8)

member of NafaQoset $(P)$, a proper absorbing subuniverse. Before we return to Step 1 (either in Step 11 or in Step 14) at least one of the sets $S_{x}^{P}$ becomes strictly smaller. It follows that there are at most $|A||V|$ returns to the first step. Finally, the last step is polynomial by Theorem III.8.

Now we show the correctness of the algorithm.

First, we observe that no solution is lost in Step 10. As the pairs $\left(x, E_{x}\right), x \in U$ are in one component of the qoset $\operatorname{Qoset}(Q)$ and the instance $Q$ is the restriction of $P$ to elements of the same component of $\operatorname{Qoset}(P)$, it follows that all the pairs $\left(x, E_{x}\right), x \in U$ lie in the same component of $\operatorname{Qoset}(P)$. Therefore, if $f: V \rightarrow A$ is a solution to $P$ such that $f(x) \in E_{x}$ for some $x \in U$, then $f(x) \in E_{x}$ for all $x \in U$ (see Proposition III.3 and the discussion bellow). But the restriction of such a function $f$ to the set $U$ would be a solution to the instance $\left.\left(\left.Q\right|_{U}\right)\right|_{\left\{E_{x}: x \in U\right\}}$, thus we would not get to this step. We have shown that in Step 10 every solution to $P$ misses all the sets $E_{x}, x \in U$, and hence we do not lose any solution when we restrict $P$ to $\mathcal{F}$.

Next, we show that if $P$ has a solution before Step 13, then the restricted instance $\left.P\right|_{\mathcal{F}}$ has a solution as well. If $f$ : $V \rightarrow A$ is a solution to $P$ such that $f(x) \notin D_{x}$ for some $x \in W$, then $f(x) \in D_{x}$ for all $x \in W$, because $\left(x, D_{x}\right)$, $x \in W$ are in the same component of $\operatorname{Qoset}(P)$ and we can use Proposition III.3 as above. In this case $f$ is a solution to the restricted instance. Now we assume that $f$ is a solution to $P$ such that $f(x) \in D_{x}$ for all $x \in W$. For each $(Q, \mathcal{E})$-strand $U$ let $g_{U}: U \rightarrow A$ be a solution to the instance $\left.\left(\left.Q\right|_{U}\right)\right|_{\left\{E_{x}: x \in U\right\}}$. Let $h: V \rightarrow A$ be the mapping satisfying $\left.h\right|_{V-W}=\left.f\right|_{V-W}$ and $\left.h\right|_{U}=\left.f\right|_{U}$ for each $(Q, \mathcal{E})$-strand $U$. We claim that this mapping is a solution to the instance $\left.P\right|_{\mathcal{F}}$.

Clearly, $h(x) \in S_{x}^{P}-\left(D_{x}-E_{x}\right)$ for every $x \in W$. 
We define $D_{x}$ for $x \in V-W$ by $D_{x}=S_{(y, x)}^{P}{ }^{+}\left[D_{y}\right]$, where $y$ is an arbitrarily chosen element of $W$. The definition of $D_{x}$ does not depend on the choice of $y$ : Let $y, y^{\prime} \in W$ and take an arbitrary $a \in S_{(y, x)}^{P}{ }^{+}\left[D_{y}\right]$. From the choice of $a$ it follows that there is $b \in D_{y}$ such that $(b, a) \in S_{(y, x)}^{P}$. Lemma III.2 provides us with an element $b^{\prime} \in A$ such that $\left(b^{\prime}, a\right) \in S_{\left(y^{\prime}, x\right)}^{P}$ and $\left(b, b^{\prime}\right) \in S_{\left(y, y^{\prime}\right)}^{P}$. The latter fact together with Proposition III.3 implies $b^{\prime} \in D_{y^{\prime}}$, therefore $a \in S_{\left(y^{\prime}, x\right)}^{P}{ }^{+}\left[D_{y^{\prime}}\right]$. We have proved the inclusion $S_{(y, x)}^{P}{ }^{+}\left[D_{y}\right] \subseteq S_{\left(y^{\prime}, x\right)}^{P}{ }^{+}\left[D_{y^{\prime}}\right]$, the opposite inclusion is proved similarly.

We put $E_{x}=D_{x}$ for $x \in V-W$. Let $\mathbf{D}_{x}$ (resp. $\mathbf{E}_{x}$ ) denote the subalgebra of $\mathbf{A}$ with universe $D_{x}$ (resp. $E_{x}$ ), $x \in V$. For any $x \in V-W$ and $y \in W$, the pair $\left(x, D_{x}\right)$ is greater than or equal to $\left(y, D_{y}\right)$ in the qoset $\operatorname{Qoset}(P)$. Since $\mathcal{D}$ is a maximal component and $x \notin W$, it follows that $D_{x}$ is outside the qoset NafaQoset $(P)$ and thus $\mathbf{D}_{x}$ has no proper absorbing subuniverse. Therefore $E_{x} \triangleleft \triangleleft \mathbf{D}_{x}$ for all $x \in V$ (for $x \in W$ it follows from the fact that $\mathcal{E}$ is the result of Algorithm 1).

Now we are ready to show that $h$ is a solution to $P$, i.e. $h$ satisfies all the constraints in $\mathcal{C}$. So, let $C=(\mathbf{x}, R) \in \mathcal{C}$, $\mathbf{x}=\left(x_{1}, \ldots, x_{n}\right)$ be an arbitrary constraint. For each $i \in V$ let $\mathbf{A}_{i}=\mathbf{D}_{x_{i}}$ and $\mathbf{B}_{i}=\mathbf{E}_{x_{i}}$, let $\mathbf{a}=\left(h\left(x_{1}\right), \ldots, h\left(x_{n}\right)\right)$, and let $L=\left\{l_{1}, \ldots, l_{k}\right\}:=\left\{i: x_{i} \in W\right\}$. By the choice of $D_{x} \mathbf{s}$, the relation $R$ is subdirect in $A_{1} \times \cdots \times A_{n}$. Since $\left.Q\right|_{\mathcal{E}}$ is 1minimal (it is the result of Algorithm 1), the projection of $C$ to $\left(x_{l_{1}}, \ldots, x_{l_{k}}\right)$ has a nonempty intersection with $B_{l_{1}} \times \cdots \times B_{l_{k}}$. By the choice of $E_{x}, x \in V-W$ it follows that the relation $R$ has a nonempty intersection with $B_{1} \times \cdots \times B_{n}$. For any $i \in L$ and $j \in\{1, \ldots, n\}-L$ we have $\left.R\right|_{(j, i)}{ }^{+}\left[B_{j}\right]=A_{i} \not \subset B_{i}$, therefore no element of $L$ is in the same $(R, B)$-strand as an element outside $L$. Moreover, $i, j \subseteq L$ are in the same $(R, B)$ strand if and only if $x_{i}, x_{j}$ are in the same $(Q, \mathcal{E})$-strand, since $\left.R\right|_{(i, j)}=S_{\left(x_{i}, x_{j}\right)}^{P}$. It follows that $\left.\left.\mathbf{a}\right|_{K} \in R\right|_{K}$ for each $(R, B)$ strand $K \subseteq L$, and the same is of course true for each $(R, B)$ strand $K \subseteq\{1,2, \ldots, n\}-L$ as $\left.f\right|_{V-W}=\left.h\right|_{V-W}$. We have checked all the assumptions of Theorem III.7, which gives us $\mathbf{a} \in R$. In other words, $h$ satisfies the constraint $C$.

From the fact that $\mathbf{A}$ is conservative it easily follows that after both Step 10 and Step 13 the restricted instance is still an instance of $\operatorname{CSP}(\operatorname{Inv} \mathbf{A})$.

Finally, we prove that $P$ satisfies the assumptions of Theorem III.8 when we get to Step 15. Note that at this point we know that no subalgebra of $\mathbf{S}_{x}^{P}$ has a proper absorbing subalgebra. Let $t$ be a cyclic term operation of the algebra $\mathbf{A}$ (guaranteed by Theorem II.2). If $t(a, a, \ldots, a, b)=a$ for some $x \in V$, $a, b \in S_{x}^{P}$, then $t(a, a, \ldots, a, b)=t(a, a, \ldots, a, b, a)=\cdots=$ $t(b, a, a, \ldots, a)$, and hence $\{a\}$ is an absorbing subuniverse of $\{a, b\}$ with respect to $t$, a contradiction. Therefore, as $\mathbf{A}$ is conservative, $t(a, a, \ldots, a, b)=b=t(b, a, a, \ldots, a)$ for any $x \in V, a, b \in S_{x}^{P}$. Now the term operation $m(x, y, z)=$ $t(x, y, y, \ldots, y, z)$ satisfies the assumptions of Theorem III.8 and the proof is concluded.

\section{Prague strategies}

This section fills the gaps in the proof of Theorem III.6.
Definition V.1. Let $P=(V, A, \mathcal{C})$ be a 1-minimal instance of the CSP. A pattern in $P$ is a tuple $\left(x_{1}, C_{1}, x_{2}, C_{2}, \ldots, C_{n-1}, x_{n}\right)$, where $x_{1}, \ldots, x_{n} \in V$ and, for every $i=1, \ldots, n-1, C_{i}$ is a constraint whose scope contains $\left\{x_{i}, x_{i+1}\right\}$. The pattern $w$ is closed with base $x$, if $x_{1}=x_{n}=x$. We define $\llbracket w \rrbracket=\left\{x_{1}, \ldots, x_{n}\right\}$.

A sequence $a_{1}, \ldots, a_{n} \in A$ is a realization of $w$ in $P$, if $\left.\left(a_{i}, a_{i+1}\right) \in C_{i}\right|_{\left(x_{i}, x_{i+1}\right)}$ for any $i \in\{1, \ldots, n-1\}$. We say that two elements $a, a^{\prime} \in A$ are connected via $w$ (in $P$ ), if there exists a realization $a=a_{1}, a_{2}, \ldots, a_{n-1}, a_{n}=a^{\prime}$ of the pattern $w$.

For two patterns $w=\left(x_{1}, C_{1}, \ldots, x_{n}\right), w^{\prime}=$ $\left(x_{1}^{\prime}, C_{1}^{\prime}, \ldots, x_{m}^{\prime}\right)$ with $x_{n}=x_{1}^{\prime}$ we define their concatenation by $w v=\left(x_{1}, C_{1}, \ldots, x_{n}, C_{1}^{\prime}, \ldots, x_{m}^{\prime}\right)$. We write $w^{k}$ for a $k$-fold concatenation of a closed pattern $w$ with itself.

Definition V.2. A 1-minimal instance $P=(V, A, \mathcal{C})$ is a Prague strategy, if for every $x \in V$, every pair of closed patterns $v, w$ in $P$ with base $x$ such that $\llbracket v \rrbracket \subseteq \llbracket w \rrbracket$, and every $a, a^{\prime} \in S_{x}^{P}$ connected via the pattern $v$ in $P$, there exists a natural number $k$ such that a is connected to $a^{\prime}$ via the pattern $w^{k}$.

First we show that every $(2,3)$-minimal instance is a Prague strategy. We need an auxiliary lemma.

Lemma V.3. Let $P=(V, A, \mathcal{C})$ be a $(2,3)$-minimal instance, let $x, x^{\prime} \in V$ and let $w=\left(x=x_{1}, C_{1}, x_{2}, \ldots, x_{n}=x^{\prime}\right)$ be a pattern. Then a is connected to $a^{\prime}$ via $w$ in $P$ for any $a, a^{\prime} \in A$ such that $\left(a, a^{\prime}\right) \in S_{\left(x, x^{\prime}\right)}^{P}$.

Proof: Using Lemma III.2 we obtain $a_{2} \in A$ such that $\left(a, a_{2}\right) \in S_{\left(x_{1}, x_{2}\right)}^{P}$ and $\left(a_{2}, a^{\prime}\right) \in S_{\left(x_{2}, x_{n}\right)}^{P}$. The element $a_{2}$ is the second (after $a$ ) element of a realization of the pattern $w$. Similarly, there exists an element $a_{3} \in A$ such that $\left(a_{2}, a_{3}\right) \in$ $S_{\left(x_{2}, x_{3}\right)}^{P}$ and $\left(a_{3}, a^{\prime}\right) \in S_{\left(x_{3}, x_{n}\right)}^{P}$. Repeated applications of this reasoning produce a realization of the pattern $w$ connecting $a$ to $a^{\prime}$.

Lemma V.4. Every $(2,3)$-minimal instance is a Prague strategy.

Proof: Let $x \in V$, let $v, w$ be closed patterns in $P$ with base $x$ such that $\llbracket v \rrbracket \subseteq \llbracket w \rrbracket$, and let $a, a^{\prime} \in S_{x}^{P}$ be elements connected via $v=\left(x_{1}, \ldots, x_{n}\right)$. Let $a=a_{1}, \ldots a_{n}=a^{\prime}$ be a realization of $v$. Since $x_{2}$ appears in $w$ there exists an initial part of $w$, say $w^{\prime}$, starting with $x$ and ending with $x_{2}$. Since $\left(a, a_{2}\right) \in S_{\left(x, x_{2}\right)}^{P}$ we use Lemma V.3 to connect $a$ to $a_{2}$ via $w^{\prime}$. Since $x_{3}$ appears in $w$ there exists $w^{\prime \prime}$ such that $w^{\prime} w^{\prime \prime}$ is an initial part of $w^{2}$ and such that $w^{\prime \prime}$ ends in $x_{3}$. Since $\left(a_{2}, a_{3}\right) \in S_{\left(x_{2}, x_{3}\right)}^{P}$ we use Lemma V.3 again to connect $a_{2}$ to $a_{3}$ via the pattern $w^{\prime \prime}$. Now $a_{1}$ and $a_{3}$ are connected via the pattern $w^{\prime} w^{\prime \prime}$. By continuing this reasoning we obtain the pattern $w^{k}$ (for some $k$ ) connecting $a$ to $a^{\prime}$.

Part (iii) of the following lemma generalizes Proposition III.3.

Lemma V.5. Let $P=(V, A, \mathcal{C})$ be a 1-minimal instance. The following are equivalent.

(i) P is a Prague strategy. 
(ii) For every $x \in V$, every pair of closed patterns $v, w$ in $P$ with base $x$ such that $\llbracket v \rrbracket \subseteq \llbracket w \rrbracket$, and every $a, a^{\prime} \in S_{x}^{P}$ connected via the pattern $v$ in $P$, there exists a natural number $m$ such that, for all $k \geq m$, the elemenents $a, a^{\prime}$ are connected via the pattern $w^{k}$;

(iii) For every two elements $(x, B),\left(x^{\prime}, B^{\prime}\right)$ in the same component of the qoset $\operatorname{Qoset}(P)$ and every constraint $C \in \mathcal{C}$ whose scope contains $\left\{x, x^{\prime}\right\}$, we have $\left.C\right|_{\left(x, x^{\prime}\right)} ^{+}[B]=$ $B^{\prime}$.

Proof: For $(i) \Longrightarrow($ ii $)$ it is clearly enough to prove the claim for $a=a^{\prime}$. To do so, we obtain (using (i)) a natural number $p$ such that $a$ is connected to $a$ via $w^{p}$. Let $b$ be an element of $A$ such that $a$ is connected to $b$ via $w$ and $b$ is connected to $a$ via $w^{p-1}$. We use the property $(i)$ for $a, b$ and the pattern $w^{p}$ to find a natural number $q$ such that $a$ is connected to $b$ via $w^{p q}$. From the facts that $a$ is connected to $a$ via $w^{p}$ and also via $w^{p q+p-1}$ (as $a$ is connected to $b$ via $w^{p q}$ and $b$ to $a$ via $w^{p-1}$ ) we get that $a$ is connected to $a$ via $w^{i p+j(p q+p-1)}$ for arbitrary $i, j$. Since $p$ and $p q+p-1$ are coprime, the claim follows.

For $(i) \Longrightarrow($ iii $)$ let $(x, B)=\left(x_{1}, B_{1}\right),\left(x_{2}, B_{2}\right)$, $\ldots,\left(x_{n}, B_{n}\right)=\left(x^{\prime}, B^{\prime}\right)=\left(x_{n+1}, B_{1}^{\prime}\right),\left(x_{n+2}, B_{2}^{\prime}\right)$, $\ldots,\left(x_{m}, B_{m}\right)=(x, B)$ be a sequence of elements of $\operatorname{Qoset}(P)$ and $C_{1}, \ldots, C_{m-1} \in \mathcal{C}$ be constraints such that $\left.C\right|_{\left(x_{i}, x_{i+1}\right)}{ }^{+}\left[B_{i}\right]=B_{i+1}$ for every $i=1, \ldots, m-1$.

Assume that there exists $a, a^{\prime} \in A$ such that $\left(a, a^{\prime}\right) \in$ $\left.C\right|_{\left(x, x^{\prime}\right)}$ and $a^{\prime} \in B^{\prime}$ while $a \notin B$. We can find an element $b \in B$ such that $b$ is connected to $a^{\prime}$ via the pattern $\left(x_{1}, C_{1}, \ldots, x_{n}\right)$. The elements $b, a$ are connected via the pattern $\left(x_{1}, C_{1}, \ldots, C_{n-1}, x_{n}, C, x_{1}\right)$, therefore, by (i), they must be connected via a power of the pattern $\left(x_{1}, C_{1}, \ldots, C_{m-1}, x_{m}\right)$, which contradicts the last property from the last paragraph. This contradiction shows that $\left.C\right|_{\left(x^{\prime}, x\right)}{ }^{+}\left[B^{\prime}\right] \subseteq B$. Similarly $\left.C\right|_{\left(x, x^{\prime}\right)}{ }^{+}[B] \subseteq B$ and the proof can be finished as in Proposition III.3.

We do not need the implication $($ iii $) \Longrightarrow(i)$ in this paper, therefore we omit the proof.

The following lemma covers the last gap.

Lemma V.6. Let $P=(V, A, \mathcal{C})$ be an instance of $\operatorname{CSP}(\operatorname{Inv} \mathbf{A})$ which is a Prague strategy and let $\mathcal{F}=\left\{\left(x, E_{x}\right): x \in W\right\}$ be a maximal component of the qoset $\mathrm{AbsQoset}(P)$. Then the restriction $Q=\left.P\right|_{\mathcal{F}}$ is a Prague strategy.

Proof: It is easy to see that, for any $x, x^{\prime} \in V$, any $B \triangleleft \mathbf{S}_{x}^{P}$ and any constraint $C$ whose scope contains $\left\{x, x^{\prime}\right\}$, the set $\left.C\right|_{\left(x, x^{\prime}\right)}{ }^{+}[B]$ is an absorbing subuniverse of $\mathbf{S}_{x^{\prime}}^{P}$ (with respect to the same term operation of $\mathbf{A})$. Therefore $\left.C\right|_{\left(x, x^{\prime}\right)}{ }^{+}\left[E_{x}\right]=$ $S_{x^{\prime}}^{P}$ whenever $x \in W$ and $x^{\prime} \in V-W$. From this fact and Lemma V.5 part (iii) it follows that $Q$ is 1-minimal.

To prove that $Q$ is a Prague strategy let $v$ and $w=(x=$ $\left.x_{1}, C_{1}, x_{2}, \ldots, C_{n-1}, x_{n}=x\right)$ be closed patterns with base $x$ such that $\llbracket v \rrbracket \subseteq \llbracket w \rrbracket$ and let $a, a^{\prime} \in S_{x}^{Q}$ be elements connected via $v$ in $Q$. Let $t$ be a $k$-ary term operation providing the absorptions $E_{x} \triangleleft \mathbf{S}_{x}^{P}$. By Lemma V.5 part (ii) we can find a natural number $m$ such that any two elements $b, b^{\prime}$, which are connected in $P$ via some closed pattern $v^{\prime}$ with base $x$ such that $\llbracket v^{\prime} \rrbracket \subseteq \llbracket w \rrbracket$, are connected via $w^{m}$.

We form a matrix with $k$ rows and $(k m(n-1)+1)$ columns. The $i$-th row is formed as follows. We find a realization (1) of the pattern $w^{(i-1) m}$ connecting $a$ to an element $b$ in $Q$. This is possible since $Q$ is 1 -minimal. (For $i=1$ we consider the empty sequence.) Then we find a realization (3) of the pattern $w^{(k-i) m}$ connecting some element $b^{\prime}$ to $a^{\prime}$ in $Q$. Finally we find a realization (2) of the pattern $w^{m}$ connecting $b$ to $b^{\prime}$ in the strategy $P$ (which is possible by the last sentence in the previous paragraph). Finally we join the realizations (1),(2),(3). When we apply the operation $t$ to the columns of this matrix, we get a realization of the pattern $w^{k m}$ connecting $a=t(a, \ldots, a)$ to $a^{\prime}=t\left(a^{\prime}, \ldots, a^{\prime}\right)$ in $Q$, which finishes the proof.

\section{PROOF OF THEOREM III. 7}

For the entire section we fix a finite idempotent Taylor algebra A.

Two absorptions can be provided by different term operations. A simple trick can unify them:

Lemma VI.1. Let $\mathbf{A}_{1}, \mathbf{A}_{2}, \mathbf{B}_{1}, \mathbf{B}_{2} \in \mathrm{V}_{\text {fin }}(\mathbf{A})$ and $\mathbf{B}_{1} \triangleleft$ $\mathbf{A}_{1}, \mathbf{B}_{2} \triangleleft \mathbf{A}_{2}$. Then there exists a term operation $t$ of $\mathbf{A}$ such that both absorptions are with respect to the operation $t$.

Proof: If $\mathbf{B}_{i}$ is an absorbing subalgebra of $\mathbf{A}_{i}$ with respect to an $n_{i}$-ary operation $t_{i}, i=1,2$, then the $n_{1} n_{2}$-ary operation defined by $t\left(a_{1}, \ldots, a_{n_{1} n_{2}}\right)=$ $t_{1}\left(t_{2}\left(a_{1}, \ldots, a_{n_{2}}\right), t_{2}\left(a_{n_{2}+1}, \ldots\right), \ldots\right)$ satisfies the conclusion.

The main tool for proving Theorem III.7 is the Absorption Theorem (Theorem III.6. in [17]). We require a definition of a linked subdirect product:

Definition VI.2. Let $R \subseteq_{S} A_{1} \times A_{2}$. We say that two elements $a, a^{\prime} \in A_{1}$ are $R$-linked via $c_{0}, c_{1}, \ldots, c_{2 n}$, if $a=c_{0}, c_{2 n}=$ $a^{\prime}$ and $\left(c_{2 i}, c_{2 i+1}\right) \in R$ and $\left(c_{2 i+2}, c_{2 i+1}\right) \in R$ for all $i=$ $0,1, \ldots, n-1$.

We say that $R$ is linked, if any two elements $a, a^{\prime} \in A_{1}$ are $R$-linked.

Theorem VI.3. [17], [18] Let $\mathbf{A}_{1}, \mathbf{A}_{2} \in \mathrm{V}_{\text {fin }}(\mathbf{T})$ be absorption free algebras and let $R \leq_{S} \mathbf{A}_{1} \times \mathbf{A}_{2}$ be linked. Then $R=A_{1} \times A_{2}$.

We will need the following consequence.

Lemma VI.4. Let $\mathbf{A}_{1}, \mathbf{A}_{2} \in \mathrm{V}_{\mathrm{fin}}(\mathbf{A})$ be absorption free algebras, let $R \leq_{S} \mathbf{A}_{1} \times \mathbf{A}_{2}$ and let $\alpha_{1}$ be a maximal congruence of $\mathbf{A}_{1}$. Then either $\left\{R^{+}[C]: C\right.$ is an $\alpha_{1}$-block $\}$ is the set of blocks of a maximal congruence $\alpha_{2}$ of $\mathbf{A}_{2}$, or $R^{+}[C]=A_{2}$ for every $\alpha_{1}$-block $C$.

Proof: If the sets $R^{+}[C]$ are disjoint, then they are blocks of an equivalence on $A_{2}$, and it is straightforward to check that this equivalence is indeed a maximal congruence of $\mathbf{A}_{2}$.

In the other case we consider the factor algebra $\mathbf{A}_{1}^{\prime}=$ $\mathbf{A}_{1} / \alpha_{1}$ and the subdirect subalgebra $R^{\prime}=\left\{\left(\left[a_{1}\right]_{\alpha_{1}}, a_{2}\right)\right.$ : 
$\left.\left(a_{1}, a_{2}\right) \in R\right\}$ of $\mathbf{A}_{1} \times \mathbf{A}_{2}$. Since $\alpha_{1}$ is maximal, the algebra $\mathbf{A}_{1}^{\prime}$ has only trivial congruences. Also, $\mathbf{A}_{1}^{\prime}$ is an absorption free algebra, because the preimage of any absorbing subalgebra $\mathbf{C} \leq \mathbf{A}^{\prime}$ is an absorbing subalgebra of $\mathbf{A}$.

We define a congruence $\sim$ on $\mathbf{A}_{1}^{\prime}$ by $\left[a_{1}\right] \sim\left[a_{2}\right]$, if $\left[a_{1}\right],\left[a_{2}\right]$ are $R^{\prime}$-linked. As not all of the sets $R^{+}[C]$ are disjoint, $\sim$ is not the diagonal congruence, therefore $\sim$ must be the full congruence, and it follows that $R^{\prime}$ is linked. By Theorem VI.3 $R^{\prime}=A_{1}^{\prime} \times A_{2}$. In other words, $R^{+}[C]=A_{2}$ for every $\alpha_{1^{-}}$ block $C$.

Links are absorbed to absorbing subuniverses:

Lemma VI.5. Let $\mathbf{A}_{1}, \mathbf{A}_{2} \in \mathrm{V}_{\text {fin }}(\mathbf{A})$, let $R \leq_{S} \mathbf{A}_{1} \times \mathbf{A}_{2}$, let $\mathbf{B}_{1} \triangleleft \mathbf{A}_{1}, \mathbf{B}_{2} \triangleleft \mathbf{A}_{2}$ and let $S=R \cap\left(B_{1} \times B_{2}\right)$ be subdirect in $B_{1} \times B_{2}$. Then every pair $b_{1}, b_{1}^{\prime} \in B_{1}$ of $R$-linked elements is also S-linked.

Proof: By Lemma VI.1 there exists a term operation $t$ such that both absorptions are with respect to $t$. Let $b_{1}, b_{1}^{\prime} \in$ $B_{1}$ be arbirary. Since $S$ is subdirect, there exist $b_{2}, b_{2}^{\prime} \in B_{2}$ such that $\left(b_{1}, b_{2}\right),\left(b_{1}^{\prime}, b_{2}^{\prime}\right) \in S$. Let $b_{1}, b_{1}^{\prime}$ be $R$-linked via $c_{0}, c_{1}, \ldots, c_{2 n}$. Now the following sequence $S$-links $b_{1}$ to $b_{1}^{\prime}$ :

$$
\begin{gathered}
b_{1}=t\left(b_{1}=c_{0}, b_{1}, \ldots, b_{1}\right), t\left(c_{1}, b_{2}, \ldots, b_{2}\right), t\left(c_{2}, b_{1}, \ldots, b_{1}\right), \\
\ldots, t\left(b_{1}^{\prime}=c_{2 n}, b_{1}, \ldots, b_{1}\right), t\left(b_{2}^{\prime}, c_{1}, b_{2}, \ldots, b_{2}\right), \ldots, \\
t\left(b_{1}^{\prime}, b_{1}^{\prime}, b_{1}, \ldots, b_{1}\right), t\left(b_{2}^{\prime}, b_{2}^{\prime}, c_{1}, b_{2}, \ldots, b_{2}\right), \ldots, \ldots, \\
t\left(b_{1}^{\prime}, \ldots, b_{1}^{\prime}\right)=b_{1}^{\prime} .
\end{gathered}
$$

A subalgebra of a conservative absorption free algebra which hits all block of a proper congruence is absorption free:

Lemma VI.6. Let $\mathbf{A}_{1} \in \mathrm{V}_{\text {fin }}(\mathbf{A})$ be a conservative absorption free algebra and let $\alpha$ be a proper congruence of $\mathbf{A}_{1}$. Then any subalgebra $\mathbf{B}$ of $\mathbf{A}_{1}$ which has a nonempty intersection with every $\alpha$-block is an absorption free algebra.

Proof: For a contradiction, consider a proper absorbing subuniverse $C$ of $\mathbf{B}$. Let $D_{1}, \ldots, D_{k}$ be all the $\alpha$-blocks whose intersections with $B$ and $C$ are equal and let $E_{1}, \ldots, E_{l}$ be the remaining $\alpha$-blocks which intersect $C$ nonempty.

We claim that, for every $m \leq l$, the set $F=D_{1} \cup \cdots \cup D_{k} \cup$ $E_{1} \cup \cdots \cup E_{m}$ is an absorbing subuniverse of $\mathbf{A}$ : Let $t$ be a term operation providing the absorption $C \triangleleft \mathbf{B}$ and let a be a tuple of elements in $A$ with all the coordinates in $F$ with the exception of, say, $a_{i}$. We take any tuple $\mathbf{b}$ such that $b_{j} \alpha a_{j}$ for all coordinates $j, b_{i} \in B-C$ and $b_{j} \in C$ for all $j \neq i$. As $B \triangleleft \mathbf{C}, t(\mathbf{b})$ is an element of $C$ and, due to conservativity, $t(\mathbf{b}) \in F$. Therefore $t(\mathbf{a}) \in F$ as this element is $\alpha$-congruent to $t(\mathbf{b})$.

For an appropriate choice of $m \leq l, F$ is a proper nonempty subset of $\mathbf{A}$ and $F \triangleleft \mathbf{A}$, a contradiction.

A subdirect product of conservative absorption free algebras is absorption free:

Lemma VI.7. Let $\mathbf{R} \leq_{S} \mathbf{A}_{1} \times \mathbf{A}_{2} \times \cdots \times \mathbf{A}_{n}$, where every $\mathbf{A}_{i} \in \mathrm{V}_{\mathrm{fin}}(\mathbf{A})$ is a conservative absorption free algebra. Then $\mathbf{R}$ is an absorption free algebra.
Proof: We take a minimal counterexample to the lemma in the following sense: We assume that the lemma holds true for every smaller $n$, and also for every $\mathbf{R}^{\prime} \leq_{S} \mathbf{A}_{1}^{\prime} \times \cdots \times$ $\mathbf{A}_{n}^{\prime}$ such that $\left|A_{i}^{\prime}\right| \leq\left|A_{i}\right|, i=1, \ldots, n$, where at least one inequality is strict. We can assume that no $A_{i}$ is one-element, otherwise we can employ the minimality assumption and use the lemma for the projection to the remaining coordinates.

Let $S$ be a proper absorbing subuniverse of $\mathbf{R}$. It is easily seen that the projection of $S$ to any coordinate $i$ is an absorbing subuniverse of $\mathbf{A}_{i}$, thus $S$ is subdirect. Let $\alpha_{1}$ be a maximal congruence of $\mathbf{A}_{1}$.

For every $i \in\{1,2, \ldots, n\}$ we have two possibilities (see Lemma VI.4):

(i) $\left\{\left.R\right|_{(1, i)}{ }^{+}[C]: C\right.$ is an $\alpha_{1}$-block $\}$ are blocks of a maximal congruence $\alpha_{i}$ of $\mathbf{A}_{i}$

(ii) $\left.R\right|_{(1, i)}{ }^{+}[C]=A_{i}$ for every $\alpha_{1}$-block $C$

Let $G$ (resp. $W$ ) denote the set of $i$ s for which the first (resp. the second) possibility takes place. By using Lemma VI.4 again, we get that $\left.R\right|_{(i, j)}{ }^{+}[C]=A_{j}$ for any $i \in G, j \in W$ and any $\alpha_{i}$-block $C$.

We take an arbitrary tuple $\left(a_{1}, \ldots, a_{n}\right) \in R$ and we aim to show that this tuple belongs to $S$ as well. The proof splits into two cases.

Assume first that for every $i \in G, \alpha_{i}$ is the diagonal congruence. Let $A_{j}^{\prime}=\left.R\right|_{(1, j)}{ }^{+}[\{a\}], j=1,2, \ldots, n$, let $R^{\prime}=R \cap\left(A_{1}^{\prime} \times \cdots \times A_{n}^{\prime}\right)$ and let $S^{\prime}=S \cap\left(A_{1}^{\prime} \times \cdots \times A_{n}^{\prime}\right)$. Note that $A_{i}^{\prime}$ is one element (for $i \in G$ ) or equal to $A_{i}$ (for $i \in W$ ), and $S^{\prime}$ absorbs $R^{\prime}$. Therefore $R^{\prime}=S^{\prime}$ (by the minimality assumption) and hence $\left(a_{1}, \ldots, a_{n}\right) \in S$.

Now assume that some $\alpha_{i}, i \in G$ is not the diagonal congruence. Take a proper subset $B$ of $A_{i}$ which contains $a_{i}$ and which intersects all $\alpha_{i}$-blocks nonempty. Let $A_{j}^{\prime}=$ $\left.R\right|_{(i, j)}{ }^{+}[B], j=1, \ldots, n$, and let $R^{\prime}, S^{\prime}$ be as in the previous paragraph. By Lemma VI.7 every $\mathbf{A}_{j}^{\prime}, j \in G$ is an absorption free algebra, and $\mathbf{A}_{j}^{\prime}=A_{j}$ for $j \in W$ is absorption free as well. Now, by the minimality assumption, $S^{\prime}=R^{\prime}$, hence $\left(a_{1}, \ldots, a_{n}\right) \in S$.

The following lemma proves a special case of Theorem III.7. Note that we do not require $\mathbf{A}_{2}$ to be conservative.

Lemma VI.8. Let $\mathbf{A}_{1}, \mathbf{A}_{2}, \mathbf{B}_{1}, \mathbf{B}_{2} \in \mathrm{V}_{\text {fin }}(\mathbf{A})$ be algebras such that $\mathbf{A}_{1}$ is conservative, $\mathbf{B}_{1} \triangleleft \triangleleft \mathbf{A}_{1}$ and $\mathbf{B}_{2} \triangleleft \triangleleft \mathbf{A}_{2}$. Let $R \leq_{S} \mathbf{A}_{1} \times \mathbf{A}_{2}$. If $R \cap\left(B_{1} \times B_{2}\right) \neq \emptyset$ and there exists $a$ pair $\left(a_{1}, b_{2}\right) \in R$ such that $a_{1} \in A_{1}-B_{1}$ and $b_{2} \in B_{2}$, then $B_{1} \times B_{2} \subseteq R$.

Proof: Let $S=R \cap\left(B_{1} \times B_{2}\right)$. As before, the projection of $S$ to the first (resp. second) coordinate is an absorbing subuniverse of $\mathbf{B}_{1}$ (resp. $\mathbf{B}_{2}$ ), and, by the assumption, $S$ is nonempty, therefore $S \leq_{S} B_{1} \times B_{2}$. Let $b_{1} \in B_{1}$ be such that $\left(b_{1}, b_{2}\right) \in R$. We define a congruence on $\mathbf{A}_{1}$ by putting $c \sim d$, if $c$ and $d$ are $R$-linked.

Let $C$ denote the set of all the elements of $B_{1}$ which are not $R$-linked to $b_{1}$. If $C$ is empty, then, by Lemma VI.5, $S$ is linked and therefore $S=B_{1} \times B_{2}$ by Theorem VI.3.

Otherwise, $C$ is a proper subuniverse of $\mathbf{B}_{1}$ and we claim that $C \triangleleft \mathbf{B}_{1}$ : Let $t$ be a term operation providing the absorption 
$B_{1} \triangleleft \mathbf{A}_{1}$ and let $\mathbf{c}$ be a tuple of elements of $B_{1}$ with all the coordinates but one, say $c_{i}$, in $C$. Let $\mathbf{d}$ be the tuple defined by $d_{i}=a_{1}$, and $d_{j}=c_{j}$ for $j \neq i$. As $d_{i} \sim c_{i}$ for all $i$ we have $t(\mathbf{c}) \sim t(\mathbf{d})$. But $t(\mathbf{d})$ lies inside $C$ (as $B_{1}$ absorbs $\mathbf{A}_{1}$ and $\mathbf{A}_{1}$ is conservative), hence also $t(\mathbf{c}) \in C$.

We have found a proper absorbing subuniverse $C$ of $\mathbf{B}_{1}$, a contradiction.

The next lemma generalizes the previous one. Recall the definition of the quasi-ordering $\preceq$ introduced in Subsection III-E.

Lemma VI.9. Let $\mathbf{A}_{1}, \ldots, \mathbf{A}_{n}, \mathbf{B}_{1}, \ldots, \mathbf{B}_{n} \in \mathrm{V}_{\text {fin }}(\mathbf{A})$ be algebras such that $\mathbf{A}_{1}, \ldots, \mathbf{A}_{n-1}$ are conservative and $\mathbf{B}_{i} \bowtie \mathbf{A}_{i}$ for all $i=1, \ldots, n$. If $\{1,2, \ldots, n-1\}$ is an $(R, B)$-strand and there exists a tuple $\left(a_{1}, a_{2}, \ldots, a_{n-1}, b_{n}\right) \in R$ such that $b_{n} \in B_{n}$ and $a_{i} \in A_{i}-B_{i}$ for some (equivalently every) $i \in\{1,2, \ldots, n-1\}$, then every tuple $\mathbf{c} \in B_{1} \times \cdots \times B_{n}$ such that $\left.\left.\mathbf{c}\right|_{\{1,2, \ldots, n-1\}} \in R\right|_{\{1,2, \ldots, n-1\}}$ belongs to $R$.

Proof: We take a minimal counterexample in the same sense as in Lemma VI.7, i.e., we assume that the lemma holds if $n$ is smaller and also if some $A_{i}$ is smaller.

We may assume that all $B_{i}$ s are at least two-element and let us also assume that if some of the algebras $\mathbf{B}_{1}, \ldots, \mathbf{B}_{n}$ has a nontrivial congruence, then $\mathbf{B}_{1}$ has a nontrivial congruence (otherwise we just change the indices).

Let $\alpha_{1}$ be a maximal congruence of $\mathbf{B}_{1}$. By applying Lemma VI.4 as in the proof of Lemma VI.7 we get that, for each $i \in\{1,2, \ldots, n-1\}$, either $\left.R\right|_{(1, i)}{ }^{+}[C]=B_{i}$ for every $\alpha_{1}$-block $C$, or $\left\{\left.R\right|_{(1, i)} ^{+}[C]: C\right.$ is an $\alpha$-block $\}$ are blocks of a maximal congruence $\alpha_{i}$ on $\mathbf{B}_{i}$.

Let $\mathbf{c} \in B_{1} \times \cdots \times B_{n}$ be an arbitrary tuple such that $\left.\left.\mathbf{c}\right|_{\{1,2, \ldots, n-1\}} \in R\right|_{\{1,2, \ldots, n-1\}}$.

If $\alpha_{1}$ is the diagonal congruence, then we put $D=$ $\left\{c_{1}\right\} \cup\left(A_{1}-B_{1}\right)$. Otherwise, we take an arbitrary $D$ such that $\left(A_{1}-B_{1}\right) \cup\left\{c_{1}\right\} \subseteq D \subsetneq A_{1}$ and $D$ intersects every $\alpha_{1}$-block nonempty. Let $A_{i}^{\prime}=\left.R\right|_{(1, i)}{ }^{+}[D], B_{i}^{\prime}=B_{i} \cap A_{i}^{\prime}$, $i=1 \ldots, n$ and $R^{\prime}=R \cap\left(A_{1}^{\prime} \times \cdots \times A_{n}^{\prime}\right)$.

For every $i \in\{1, \ldots, n-1\}, B_{i}^{\prime}$ is an absorption free algebra, either because $B_{i}^{\prime}$ is a singleton, or $B_{i}^{\prime}$ intersects every $\alpha_{i}$ block nonempty and we can apply Lemma VI.6. From Lemma VI.8 it follows that $B_{1} \times\left. B_{n} \subseteq R\right|_{(1, n)}$, therefore $B_{n}^{\prime}=$ $B_{n}$, in particular, $\left(a_{1}, a_{2}, \ldots, a_{n-1}, b_{n}\right) \in R^{\prime}$. Obviously $B_{i}^{\prime}$ is an absorbing sublagebra of $\mathbf{A}_{i}^{\prime}$ for every $i=1, \ldots, n$. Now $\mathbf{c} \in R^{\prime}(\subseteq R)$ follows from the miniminality of our counterexample.

We are ready to prove Theorem III.7.

Theorem VI.10. Let $\mathbf{A}_{1}, \ldots, \mathbf{A}_{n}, \mathbf{B}_{1}, \ldots, \mathbf{B}_{n} \in \mathrm{V}_{\text {fin }}(\mathbf{A})$ be conservative algebras such that $\mathbf{B}_{i} \triangleleft \mathbf{A}_{i}$ for all $i=1, \ldots, n$, let $R \leq_{S} \mathbf{A}_{1} \times \cdots \times \mathbf{A}_{n}$ and assume that $R \cap\left(B_{1} \times \ldots, B_{n}\right) \neq$ $\emptyset$. Then a tuple $\mathbf{a} \in B_{1} \times \cdots \times B_{n}$ belongs to $R$ whenever $\left.\left.\mathbf{a}\right|_{K} \in R\right|_{K}$ for each $(R, B)$-strand $K$.

Proof: We again use the minimality assumption, i.e., we assume that the theorem holds if $n$ is smaller, or if some $A_{i}$ is smaller. We can assume that there are at least two $(R, B)$-strands and that $\left|B_{i}\right|>1$ for all $i=1, \ldots, n$. Let $\mathbf{a} \in B_{1} \times \cdots \times B_{n}$ be a tuple such that $\left.\left.\mathbf{a}\right|_{K} \in R\right|_{K}$ for each $(R, B)$-strand $K$, but $\mathbf{a} \notin R$. Note that $\left.\left.\mathbf{a}\right|_{L} \in R\right|_{L}$ for every proper subset $L$ of $\{1,2, \ldots, n\}$, because of the minimality assumption - we can apply the theorem to $\left.R\right|_{L}$.

Let $D$ be a minimal $(R, B)$-strand and let $l \notin D$. Since $l \npreceq D$, there exists a tuple $\mathbf{c} \in R$ such that $c_{l} \in B_{l}$ and $c_{i} \notin B_{i}$ for all $i \in D$. Let $E=\left\{i \in\{1, \ldots, n\}: c_{i} \notin B_{i}\right\}-D$ and $F=\left\{i \in\{1, \ldots, n\}: c_{i} \in B_{i}\right\}$. Clearly, $E$ and $F$ are unions of $(R, B)$-strands.

Our aim now is to find a tuple $\mathbf{c}^{\prime} \in R$ such that $c_{i}^{\prime} \in A_{i}-B_{i}$ for all $i \in D$, and $c_{i}^{\prime} \in B_{i}$ for all $i \notin D$. If $E=\emptyset$, we can take $\mathbf{c}^{\prime}=\mathbf{c}$, so suppose otherwise. We consider the following subset of $R$ :

$$
R^{\prime}=\left\{\mathbf{b} \in R: b_{i} \in\left\{a_{i}\right\} \cup\left(A_{i}-B_{i}\right) \text { for all } i \in D\right\} .
$$

For all $i \in\{1, \ldots, n\}$, let $A_{i}^{\prime}=\left.R^{\prime}\right|_{\{i\}}$. Let $B_{i}^{\prime}=\left\{a_{i}\right\}$ for all $i \in D$, and $B_{i}^{\prime}=B_{i}$ for $i \notin D$.

We have $B_{i}^{\prime} \subseteq A_{i}$ for every $i \notin D$ : for any $b \in B_{i}$ we apply the theorem for $\left.R\right|_{D \cup\{i\}}$ to obtain a tuple $\mathbf{e} \in R$ such that $e_{i}=b$ and $e_{j}=d_{j}$ for every $j \in D$.

We know that $\left.\left.\mathbf{a}\right|_{D \cup E} \in R\right|_{D \cup E}$, therefore $\left.\left.a\right|_{E} \in R^{\prime}\right|_{E}$. Similarly, $\left.\left.a\right|_{F} \in R^{\prime}\right|_{F}$.

Observe that any $i \in E, j \in F$ are in different $\left(R^{\prime}, B^{\prime}\right)$ strands, since $\mathbf{c} \in R^{\prime}$. Therefore, the theorem, used for $R^{\prime}$ and the minimal absorbing subuniverses $B_{i}^{\prime}$ of $A_{i}^{\prime}$, proves a $\left.\right|_{E \cup F} \in$ $\left.R^{\prime}\right|_{E \cup F}$. Let $\mathbf{c}^{\prime}$ be a tuple from $R^{\prime}$ with $\left.\mathbf{c}^{\prime}\right|_{E \cup F}=\left.\mathbf{a}\right|_{E \cup F}$. The tuple $\mathbf{c}^{\prime}$ cannot be equal to $\mathbf{a}$ as $\mathbf{a} \notin R$, therefore $c_{i}^{\prime} \in A_{i}-B_{i}$ for all $i \in D, c_{i}^{\prime} \in B_{i}$ for $i \notin D$.

Now, when we have the sought after tuple $\mathbf{c}^{\prime}$, we can finish the proof by applying Lemma VI.9 for the following choice: $n^{\prime}=|D|+1 ; \mathbf{A}_{i}^{\prime}=\mathbf{A}_{d_{i}}$ and $\mathbf{B}_{i}^{\prime}=\mathbf{B}_{d_{i}}$ for $i=1, \ldots, l$, where $D=\left\{d_{1}, \ldots, d_{l}\right\} ; \mathbf{A}_{n^{\prime}}^{\prime}=\left.R\right|_{E \cup F} ; \mathbf{B}_{n^{\prime}}^{\prime}=\left.S\right|_{E \cup F}$, where $S=\left\{\mathbf{b} \in R: b_{i} \in B_{i}\right.$ for all $\left.i \in E \cup F\right\}$; and $\left(a_{1}^{\prime}, a_{2}^{\prime}, \ldots, a_{n^{\prime}-1}^{\prime}, b_{n}^{\prime}\right)=\left(c_{d_{1}}, c_{d_{2}}, \ldots, c_{d_{l}},\left.\mathbf{c}^{\prime}\right|_{E \cup F}\right)$. All the assumptions are satisfied, the only nontrivial fact is that $\mathbf{B}_{n^{\prime}}^{\prime}$ is absorption free and this follows from Lemma VI.7.

\section{CONCLUSION}

We have presented a new, simple algorithm for solving tractable CSPs over conservative languages. We believe that this simplification can help in the final attack on the dichotomy conjecture.

No effort has been made to optimize the algorithm, we has not computed its time complexity and we has not compared the complexity with the algorithm of Bulatov. This can be a topic of further research.

We note that some reductions can be done using a trick from [26], it would be interesting to see whether this trick can improve the running time.

\section{ACKNOWLEDGMENT}

I would like to thank Marcin Kozik, Miklós Maróti and Ralph McKenzie for helpful discussions. The research was supported by the Grant Agency of the Czech Republic, grant 201/09/P223 and by the Ministry of Education of the Czech Republic, grants MSM 0021620839 and MEB 040915. 


\section{REFERENCES}

[1] T. Feder and M. Y. Vardi, "The computational structure of monotone monadic SNP and constraint satisfaction: a study through Datalog and group theory," SIAM J. Comput., vol. 28, no. 1, pp. 57-104 (electronic), 1999. [Online]. Available: http://dx.doi.org/10.1137/S0097539794266766

[2] P. Jeavons, D. Cohen, and M. Gyssens, "Closure properties of constraints," J. ACM, vol. 44, no. 4, pp. 527-548, 1997. [Online]. Available: http://dx.doi.org/10.1145/263867.263489

[3] A. A. Bulatov, A. A. Krokhin, and P. Jeavons, "Constraint satisfaction problems and finite algebras," in Automata, languages and programming (Geneva, 2000), ser. Lecture Notes in Comput. Sci. Berlin: Springer, 2000, vol. 1853, pp. 272-282.

[4] A. Bulatov, P. Jeavons, and A. Krokhin, "Classifying the complexity of constraints using finite algebras," SIAM J. Comput., vol. 34, no. 3, pp. 720-742 (electronic), 2005. [Online]. Available: http://dx.doi.org/10.1137/S0097539700376676

[5] A. Bulatov, "Mal'tsev constraints are tractable," Computing Laboratory, University of Oxford, Oxford, UK, Tech. Rep. PRG-RR-02-05, 2002.

[6] A. Bulatov and V. Dalmau, "A simple algorithm for $\mathrm{Mal}^{\prime}$ tsev constraints," SIAM J. Comput., vol. 36, no. 1, pp. 16-27 (electronic), 2006. [Online]. Available: http://dx.doi.org/10.1137/050628957

[7] V. Dalmau, "Generalized majority-minority operations are tractable," Log. Methods Comput. Sci., vol. 2, no. 4, pp. 4:1, 14, 2006.

[8] P. M. Idziak, P. Markovic, R. McKenzie, M. Valeriote, and R. Willard, "Tractability and learnability arising from algebras with few subpowers," in LICS. IEEE Computer Society, 2007, pp. 213-224.

[9] L. Barto and M. Kozik, "Constraint satisfaction problems of bounded width," in FOCS'09: Proceedings of the 50th Symposium on Foundations of Computer Science, 2009, pp. 595-603.

[10] - "Constraint satisfaction problems solvable by local consistency methods," in preparation.

[11] A. A. Bulatov, "A dichotomy theorem for constraint satisfaction problems on a 3-element set," J. ACM, vol. 53, no. 1, pp. 66-120 (electronic), 2006. [Online]. Available: http://dx.doi.org/10.1145/1120582.1120584

[12] T. J. Schaefer, "The complexity of satisfiability problems," in Conference Record of the Tenth Annual ACM Symposium on Theory of Computing (San Diego, Calif., 1978). New York: ACM, 1978, pp. 216-226.

[13] A. A. Bulatov, "Tractable conservative constraint satisfaction problems," Logic in Computer Science, Symposium on, vol. 0, p. 321, 2003.

[14] L. Barto, M. Kozik, and T. Niven, "Graphs, polymorphisms and the complexity of homomorphism problems," in STOC '08: Proceedings of the 40th annual ACM symposium on Theory of computing. New York, NY, USA: ACM, 2008, pp. 789-796.

[15] _ "The CSP dichotomy holds for digraphs with no sources and no sinks (a positive answer to a conjecture of Bang-Jensen and Hell)," SIAM J. Comput., vol. 38, no. 5, pp. 1782-1802, 2008/09. [Online]. Available: http://dx.doi.org/10.1137/070708093

[16] L. Barto and M. Kozik, "Congruence distributivity implies bounded width," SIAM Journal on Computing, vol. 39, no. 4, pp. 1531-1542, 2009. [Online]. Available: http://link.aip.org/link/?SMJ/39/1531/1

[17] — "New conditions for Taylor varieties and CSP," Logic in Computer Science, Symposium on, vol. 0, pp. 100-109, 2010.

[18] _ _Absorbing subalgebras, cyclic terms and the constraint satisfaction problem," submitted.

[19] T. Feder, P. Hell, and J. Huang, "List homomorphisms and circular arc graphs," Combinatorica, vol. 19, pp. 487-505, 1999.

[20] - "Bi-arc graphs and the complexity of list homomorphisms," $J$ Graph Theory, vol. 42, pp. 61-80, January 2003. [Online]. Available: http://portal.acm.org/citation.cfm?id=1380681.1380685

[21] P. Hell and A. Rafiey, "The dichotomy of list homomorphisms for digraphs," CoRR, vol. abs/1004.2908, 2010.

[22] L. Egri, A. Krokhin, B. Larose, and P. Tesson, "The complexity of the list homomorphism problem for graphs," in 27th International Symposium on Theoretical Aspects of Computer Science (STACS 2010), ser. Leibniz International Proceedings in Informatics (LIPIcs), J.-Y. Marion and T. Schwentick, Eds., vol. 5. Dagstuhl, Germany: Schloss Dagstuhl-Leibniz-Zentrum fuer Informatik, 2010, pp. 335-346. [Online]. Available: http://drops.dagstuhl.de/opus/volltexte/2010/2467

[23] R. Takhanov, "A dichotomy theorem for the general minimum cost homomorphism problem," in 27th International Symposium on Theoretical Aspects of Computer Science (STACS 2010), ser. Leibniz
International Proceedings in Informatics (LIPIcs), J.-Y. Marion and T. Schwentick, Eds., vol. 5. Dagstuhl, Germany: Schloss DagstuhlLeibniz-Zentrum fuer Informatik, 2010, pp. 657-668. [Online]. Available: http://drops.dagstuhl.de/opus/volltexte/2010/2493

[24] V. Kolmogorov and S. Zivny, "The complexity of conservative finitevalued csps," CoRR, vol. abs/1008.1555, 2010

[25] M. Maróti and R. McKenzie, "Existence theorems for weakly symmetric operations," Algebra Universalis, vol. 59, no. 3-4, pp. 463-489, 2008 [Online]. Available: http://dx.doi.org/10.1007/s00012-008-2122-9

[26] M. Maróti, "Tree on top of maltsev," preprint. 